\title{
Micelles-A Brief Insight
}

\author{
Nida Tabassum Khan* \\ Department of Biotechnology, Faculty of Life Sciences and Informatics, Balochistan University of Information \\ Technology Engineering and Management Sciences, (BUITEMS), Quetta, Pakistan
}

*Corresponding Author: Nida Tabassum Khan, Department of Biotechnology, Faculty of Life Sciences and Informatics, Balochistan University of Information Technology Engineering and Management Sciences, (BUITEMS), Quetta, Pakistan, Email: nidatabassumkhan@yahoo.com

\begin{abstract}
Nano scale systems have been developed in the arena of nanomedicine for the identification, preclusion and treatment of various diseases. Such systems includes polymeric conjugate molecules like micelles that display effective potential to be used in therapeutics. Micelle is an aggregate of molecules that forms a colloidal spherical structure exhibiting physical compatibility with other molecules making it suitable as a pharmaceutical agent. Polymeric self- assembling micelles are commonly considered as convenient nano- carriers in diagnostic, imaging, delivery systems etc.
\end{abstract}

Keywords: Polymeric micelles; passive targeting; Dendrimers; Liposomes; Tumour targeting.

\section{INTRODUCTION}

Micelle is formed by the aggregation of several atoms, electrically charged molecules etc, attaining a spherical assembly which is hydrophobic from the inside and hydrophilic from the outer surface enabling its interaction with water molecule $[1,2]$. Micelles exhibits high degree of compatibility with other biological molecules [3]. Thus displayed high stability with the potential to solubilise weakly soluble drugs $[4,5]$.

\section{Formation OF MiCELLES}

Micelles are formed by the conjugation of lipid molecules that assembles itself in a spherical form dispersed in an aqueous solution [6]. The driving force for this arrangement is the hydrophobic interactions that the molecules experience [7]. Micelles is formed only when the concentration of the amphiphilic molecule reaches a given concentration called the critical micelle concentration [8,9]. Micelle formation depends on the tendency of the hydrophobic tails to avoid contacts with water and repulsion among the hydrophilic heads that lead to the destabilization of aggregation process [10,11].

\subsection{Size of Micelles}

Micelles usually range from $2 \mathrm{~nm}$ to $20 \mathrm{~nm}$ in size, depending on its composition and concentration [12].

\subsection{Types of Micelles}

Based on the conditions in which micelles are formed, it has different types such as:

- Block copolymer Micelles: It is formed by the self-assembly of either oppositely charged copolymers oramphiphilic molecules in aqueous medium [13]. The core of the micelles is hydrophobic however the hydrophilic blocks forms the outer portion [14]. Block copolymer micelles aids in the passive targeting of cancerous/ inflamed tissues due to enhanced permeation and retention effect $[15,16]$.

- Inverse/reverse Micelles: Hydrophilic groups are sequestered in the core of micelles while the hydrophobic groups protrude away from the centre [17].

- Super Micelles: It is the supramolecular assembly of many individual block copolymers by the formation of strong covalent bonds however within the micelles structure, components are loosely held together by electrostatic or hydrogen bonds $[18,19]$.

\subsection{Applications of Micelles}

Micelles surface could be modified to improve its specificity for the fabrication of multifunctional micelles which serves as an effective nano carrier in drug delivery systems 
[20]. Some of the applications of micelles is discussed below:

- Water Insoluble Drugs: Self-assembly of compatible amphiphilic block copolymers forms nano sized spherical structures in aqueous environments [21]. Hydrophobic portion forms the micelles core thus acting as a pool for non-polar drugs, while the superficial hydrophilic shell protects micelle from damage [22, 23].

- Cancer Treatment: Recently, nanoscale therapeutic systems such as polymeric conjugates, liposomes, dendrimers etc are employed for cancer diagnosis and treatment [24].

- Delivery of Drugs to the Brain: Drugs used for the treatment of brain disorders, tumours etc come across a restricted transport because of "blood brain barrier" [25]. Polymeric micelles modified with ligands/antibodies molecules are proficient in passing the brain endothelial cells in order to achieve maximal antitumor efficacy [26].

- Delivery of Imaging Agents: Micelles can be used as carriers for imaging agents such as amphiphilic PEO- lipid conjugates which were used for visualization of local lymphatic chain in rabbits [27].

\section{CONCLUSION}

Overall micelles play a central role in nano medicine drug delivery, tumour treatment, stimulated discharge of therapeutics etc. Polymeric micelles are emerging as multifunctional nano therapeutics for cancer diagnosis and treatment.

\section{REFERENCES}

[1] Tanford, C. (1980). The hydrophobic effect: formation of micelles and biological membranes $2 \mathrm{~d}$ ed. J. Wiley.

[2] Chapman, D., Jones, M. N., \& Jones, M. N. (1995). Micelles, monolayers, and biomembranes. Wiley-Liss.

[3] Zana, R., \& Kaler, E. W. (2007). Giant micelles: properties and applications. CRC press.

[4] Aliabadi, H. M., \& Lavasanifar, A. (2006). Polymeric micelles for drug delivery. Expert opinion on drug delivery, 3(1), 139-162.

[5] Kwon, G. S. (2003). Polymeric micelles for delivery of poorly water-soluble compounds. Critical Reviews ${ }^{\mathrm{TM}}$ in Therapeutic Drug Carrier Systems, 20(5).
[6] Lukyanov, A. N., \& Torchilin, V. P. (2004). Micelles from lipid derivatives of water-soluble polymers as delivery systems for poorly soluble drugs. Advanced drug delivery reviews, 56(9), 1273-1289.

[7] Zhang, L., Chan, J. M., Gu, F. X., Rhee, J. W., Wang, A. Z., Radovic-Moreno, A. F., ... \& Farokhzad, O. C. (2008). Self-assembled lipidpolymer hybrid nanoparticles: a robust drug delivery platform. ACS nano, 2(8), 1696-1702.

[8] Mukerjee, P., \& Mysels, K. J. (1971). Critical micelle concentrations of aqueous surfactant systems (No. NSRDS-NBS-36). National Standard reference data system.

[9] Leibler, L., Orland, H., \& Wheeler, J. C. (1983). Theory of critical micelle concentration for solutions of block copolymers. The Journal of chemical physics, 79(7), 3550-3557.

[10] Phillips, J. N. (1955). The energetics of micelle formation. Transactions of the Faraday Society, 51, 561-569.

[11] Blesic, M., Marques, M. H., Plechkova, N. V., Seddon, K. R., Rebelo, L. P. N., \& Lopes, A. (2007). Self-aggregation of ionic liquids: micelle formation in aqueous solution. Green Chemistry, 9(5), 481-490.

[12] Nelson, P. H., Rutledge, G. C., \& Hatton, T. A. (1997). On the size and shape of self-assembled micelles. The Journal of chemical physics, 107(24), 10777-10781.

[13] Gohy, J. F. (2005). Block copolymer micelles. In Block copolymers II (pp. 65-136). Springer, Berlin, Heidelberg.

[14] Gaucher, G., Deferens, M. H., Sant, V. P., Kang, N., Maysinger, D., \& Leroux, J. C. (2005). Block copolymer micelles: preparation, characterization and application in drug delivery. Journal of controlled release, 109(1-3), 169-188.

[15] Noolandi, J., \& Hong, K. M. (1983). Theory of block copolymer micelles in solution. Macromolecules, 16(9), 1443-1448.

[16] Kataoka, K., Harada, A., \& Nagasaki, Y. (2012). Block copolymer micelles for drug delivery: design, characterization and biological significance. Advanced drug delivery reviews, 64, 37-48.

[17] Pileni, M. P. (1989). Structure and reactivity in reverse micelles.

[18] McConnell, G. A., Lin, M. Y., \& Gast, A. P. (1995). Long range order in polymeric micelles under steady shear. Macromolecules, 28(20), 6754-6764.

[19] Wurm, F., König, H. M., Hilf, S., \& Kilbinger, A. F. (2008). Janus micelles induced by olefin metathesis. Journal of the American Chemical Society, 130(18), 5876-5877.

[20] Fendler, J. H. (1982). Membrane mimetic chemistry: characterizations and applications of micelles, microemulsions, monolayers, 
bilayers, vesicles, host-guest systems, and polyions (p. 522). New York: Wiley.

[21] Lukyanov, A. N., \& Torchilin, V. P. (2004). Micelles from lipid derivatives of water-soluble polymers as delivery systems for poorly soluble drugs. Advanced drug delivery reviews, 56(9), 1273-1289.

[22] Kalepu, S., \& Nekkanti, V. (2015). Insoluble drug delivery strategies: review of recent advances and business prospects. Acta Pharmaceutica Sinica B, 5(5), 442-453.

[23] Sahoo, S. K., \& Labhasetwar, V. (2003). Nanotech approaches to drug delivery and imaging. Drug discovery today, 8(24), 11121120.

[24] Misra, R., Acharya, S., \& Sahoo, S. K. (2010). Cancer nanotechnology: application of nanotechnology in cancer therapy. Drug discovery today, 15(19-20), 842-850.

[25] Zhang, L., Gu, F. X., Chan, J. M., Wang, A. Z., Langer, R. S., \& Farokhzad, O. C. (2008). Nanoparticles in medicine: therapeutic applications and developments. Clinical pharmacology \& therapeutics, 83(5), 761-769.

[26] Kabanov, A. V., \& Batrakova, E. V. (2004). New technologies for drug delivery across the blood brain barrier. Current pharmaceutical design, 10(12), 1355-1363.

[27] Janib, S. M., Moses, A. S., \& MacKay, J. A. (2010). Imaging and drug delivery using theranostic nanoparticles. Advanced drug delivery reviews, 62(11), 1052-1063.

Citation: Nida Tabassum Khan, Micelles-A Brief Insight. ARC Journal of Dermatology. 2019; 4(1):12-14. doi:dx.doi.org/10.20431/2456-0022.0401004.

Copyright: (C) 2019 Authors. This is an open-access article distributed under the terms of the Creative Commons Attribution License, which permits unrestricted use, distribution, and reproduction in any medium, provided the original author and source are credited. 